\title{
Gromov hyperbolic equivalence of the hyperbolic and quasihyperbolic metrics in Denjoy domains
}

\author{
Peter Hästö, Ana Portilla, José M. Rodríguez and Eva Tourís
}

\begin{abstract}
In this article we investigate the Gromov hyperbolicity of Denjoy domains equipped with the hyperbolic or the quasihyperbolic metric. We first prove the existence of suitable families of quasigeodesics. The main result shows that a Denjoy domain is Gromov hyperbolic with respect to the hyperbolic metric if and only it is Gromov hyperbolic with respect to the quasihyperbolic metric. Using these tools we give a characterization in terms of Euclidean distances of when the domains are Gromov hyperbolic. We also give several concrete examples of families of domains satisfying the criteria of the theorems.
\end{abstract}

\section{Introduction}

In the 1980s M. Gromov, cf. [11], introduced a notion of abstract hyperbolic spaces, which have thereafter been studied and developed by many authors, e.g. $[\mathbf{7}, \mathbf{8}, \mathbf{1 6}, \mathbf{2 5}]$. Initially, the research was mainly centered on hyperbolic group theory; lately researchers have shown an increasing interest in more direct studies of spaces endowed with metrics used in geometric function theory, e.g. $[\mathbf{2}, \mathbf{4}, \mathbf{6}, \mathbf{1 2}, \mathbf{1 4}, \mathbf{1 5}]$. One of the primary questions is naturally whether a metric space $(X, d)$ is hyperbolic in the sense of Gromov or not. A classical example of a Gromov hyperbolic space is a Riemannian manifold with sectional curvature $K \leqslant-k^{2}<0$.

Gromov hyperbolicity of the quasihyperbolic metric was studied in M. Bonk, J. Heinonen and P. Koskela [6] and a geometric characterization of when a quasihyperbolic space is Gromov hyperbolic was given by Z. Balogh and S. Buckley in [2] in terms of a slice condition. The Gromov hyperbolicity of the Poincaré hyperbolic metric is not as well understood, although several intrinsic results were obtained in $[\mathbf{1}]$ and $[\mathbf{1 7}]-[\mathbf{2 3}]$.

Since there is a geometric characterization of when a domain with the quasihyperbolic metric is Gromov hyperbolic, and since Beardon and Pommerenke [3] have related the hyperbolic and quasihyperbolic densities by a quantity with a nice geometric interpretation (see Definition 3.1), it is natural to try to approach the Gromov hyperbolicity of the hyperbolic metric from the corresponding results for the quasihyperbolic metric. An immediate question is whether there is any difference between the metrics in this respect, i.e. whether there exists a domain such that one of these metrics is Gromov hyperbolic and the other is not; if such domains exists, the next problem is to describe this class of domains.

Denjoy domains form a fairly large class of domains, and since the Gromov hyperbolicity of the hyperbolic metric was understood to some extent in this case, we considered this a good place to start to understand the differences between the two metrics. The result of this investigation, presented in this paper, was a surprise: we found that in the class of

2000 Mathematics Subject Classification 30F45 (primary), 53C23, 30C99 (secondary).

PH was supported in part by the Academy of Finland and the Emil Aaltonen Foundation. AP, JR and ET were supported in part by three grants from M.E.C. (MTM 2006-13000-C03-02, MTM 2006-11976 and MTM 2007-30904-E) and a grant from U.C.III M./C.A.M. (CCG07-UC3M/ESP-3339), Spain. 
Denjoy domains there is no difference between the metrics in terms of Gromov hyperbolicity (Theorem 4.6). Whether or not this is true in general domains remains an open question.

This paper is a continuation of $[\mathbf{1 3}]$, by the authors and H. Lindén. The main innovation compared to that study is that we now work with quasigeodesics instead of geodesics. The tools that are needed for this are developed in Section 3. As already stated, the main result is that a Denjoy domain is Gromov hyperbolic with respect to the hyperbolic metric if and only if it is Gromov hyperbolic with respect to the quasihyperbolic one. This result is proved in Section 4. Another consequence of our techniques is a simple characterization of when a Denjoy domain is Gromov hyperbolic with either of the aforementioned metrics, based only on the Euclidean lengths of the boundary segments (Theorem 5.2). In Denjoy domains this characterization is much simpler to use than the general results of $[\mathbf{2}, \mathbf{6}]$; as a demonstration we give in Section 5 several concrete classes of domains which are, or are not, Gromov hyperbolic.

\section{Definitions and notation}

By $\mathbb{H}$ we denote the upper half plane, $\{z \in \mathbb{C}: \operatorname{Im} z>0\}$, and by $\mathbb{D}$ the unit disk $\{z \in$ $\mathbb{C}:|z|<1\}$. For $D \subset \mathbb{C}$ we denote by $\partial D$ and $\bar{D}$ its boundary and closure, respectively. For $z \in D \subsetneq \mathbb{C}$ we denote by $\delta_{D}(z)$ the distance to the boundary, $\min _{a \in \partial D}|z-a|$. Finally, we denote by $c$ and $C$ generic constants which can change their value from line to line and even in the same line. We say that an inequality holds quantitatively, if it holds with a constant depending only on the constants in the assumptions.

Recall that a domain $\Omega \subset \mathbb{C}$ is said to be of hyperbolic type if it has at least two finite boundary points. The universal cover of such domain is the unit disk $\mathbb{D}$. In $\Omega$ we can define the Poincaré or hyperbolic metric, i.e. the metric obtained by pulling back the density $d s=$ $2|d z| /\left(1-|z|^{2}\right)$ of the unit disk. The quasihyperbolic metric is the distance induced by the density $1 / \delta_{\Omega}(z)$. By $k_{\Omega}$ and $h_{\Omega}$ we denote the quasihyperbolic and hyperbolic distance in $\Omega$, respectively.

Length (of a curve) will be denoted by the symbol $\ell_{d, \Omega}$, where $d$ is the metric with respect to which length is measured. The subscript "Eucl" is used to denote the length with respect to the Euclidean metric. Also, as most of the proofs apply to both the quasihyperbolic and the Poincaré metrics, we use the symbol $\kappa$ as a "dummy metric" symbol, which stands for either $k$ or $h$. We denote by $\lambda_{\Omega}$ the density of the hyperbolic metric in $\Omega$. It is well known that for every domain $\Omega$

$$
\lambda_{\Omega}(z) \leqslant \frac{2}{\delta_{\Omega}(z)} \quad \forall z \in \Omega, \quad \ell_{h, \Omega}(\gamma) \leqslant 2 \ell_{k, \Omega}(\gamma) \quad \forall \gamma \subset \Omega,
$$

and that for all domains $\Omega_{1} \subset \Omega_{2}$ we have $\lambda_{\Omega_{1}}(z) \geqslant \lambda_{\Omega_{2}}(z)$ for every $z \in \Omega_{1}$.

A geodesic metric space $(X, d)$ is said to be Gromov $\delta$-hyperbolic, if

$$
d(w,[x, z] \cup[z, y]) \leqslant \delta
$$

for every $x, y, z \in X$, corresponding geodesic segments $[x, y],[y, z]$ and $[x, z]$, and some $w \in$ $[x, y]$. If this inequality holds, we also say that the geodesic triangle is $\delta$-thin, so Gromov hyperbolicity can be reformulated as requiring that all geodesic triangles are thin.

A Denjoy domain $\Omega \subset \mathbb{C}$ is a domain whose boundary is contained in the real axis. Hence, it satisfies $\Omega \cap \mathbb{R}=\cup_{n \in \Lambda}\left(a_{n}, b_{n}\right)$, where $\Lambda$ is a countable index set, $\left\{\left(a_{n}, b_{n}\right)\right\}_{n \in \Lambda}$ are pair-wise disjoint, and $a_{n}, b_{n} \in \mathbb{R} \cup\{-\infty, \infty\}$. In order to study Gromov hyperbolicity, we may restrict ourselves to the case where $\Lambda$ is countably infinite, since if $\Lambda$ is finite then $h_{\Omega}$ and $k_{\Omega}$ are easily seen to be Gromov hyperbolic by [13, Proposition 3.5].

Let $\Omega$ be a Denjoy domain. Then we have $\Omega \cap \mathbb{R}=\cup_{n \geqslant 0}\left(a_{n}, b_{n}\right)$ for some suitable intervals. We say that a curve in $\Omega$ is a fundamental geodesic if it is a geodesic joining $\left(a_{0}, b_{0}\right)$ and $\left(a_{n}, b_{n}\right), n>0$, which is contained in the closed halfplane $\overline{\mathbb{H}}=\{z \in \mathbb{C}: \operatorname{Im} z \geqslant 0\}$. 


\section{Geodesics and quasigeodesics in Denjoy domains}

One of the main obstacles when we work with the hyperbolic or the quasihyperbolic metric is that we have scant information on the location of geodesics. We start by studying the behavior of quasigeodesics in Denjoy domains with the hyperbolic or quasihyperbolic metric in this section; this will inform us also on geodesics, as we will see.

Definition 3.1. For every domain $\Omega \subset \mathbb{C}$ of hyperbolic type define $\beta_{\Omega}: \Omega \rightarrow \mathbb{R}$ as the function

$$
\beta_{\Omega}(z):=\min \left\{|\log | \frac{z-a}{b-a}||: a, b \in \partial \Omega,|z-a|=\delta_{\Omega}(z)\right\} .
$$

This function was introduced by Beardon and Pommerenke [3] who showed that it provides the connection between the densities of the hyperbolic and the quasihyperbolic metrics.

Theorem $3.2[\mathbf{3}$, Theorem 1]. For every domain $\Omega \subset \mathbb{C}$ of hyperbolic type and for every $z \in \Omega$, we have that

$$
2^{-3 / 2} \leqslant \lambda_{\Omega}(z) \delta_{\Omega}(z)\left(k_{0}+\beta_{\Omega}(z)\right) \leqslant \pi / 4
$$

where $k_{0}=4+\log (3+2 \sqrt{2})$.

Lemma 3.3. Let us consider a Denjoy domain $\Omega, z_{0} \in \Omega \cap \overline{\mathbb{H}}$, a curve $\eta$ with Euclidean length $r$ starting at $z_{0}$, and $\sigma:=\left[z_{0}, z_{0}+i r\right]$. Then $\ell_{h, \Omega}(\sigma) \leqslant c_{0} \ell_{h, \Omega}(\eta)$, with $c_{0}:=\pi k_{0} /\left(k_{0}-\right.$ $\log \sqrt{2}$ ), where $k_{0}$ is the constant in Theorem 3.2 .

Proof. Consider the curve $\eta$ parameterized by Euclidean arc-length starting at $z_{0}$. For $t \in[0, r]$ define $z_{t}:=z_{0}+i t$, and consider $a, b \in \partial \Omega$ such that $\left|z_{t}-a\right|=\delta_{\Omega}\left(z_{t}\right)$ and

$$
\beta_{\Omega}\left(z_{t}\right)=|\log | \frac{z_{t}-a}{b-a}|| .
$$

Using the triangle inequality and elementary estimates we see that

$$
|\eta(t)-a| \leqslant\left|\eta(t)-z_{0}\right|+\left|z_{0}-a\right| \leqslant\left|z_{t}-z_{0}\right|+\left|z_{0}-a\right| \leqslant \sqrt{2}\left|z_{t}-a\right| .
$$

Since the function $x \mapsto x\left(k_{0}+|\log (x / c)|\right)$ is increasing on $(0, \infty)$ for any $c>0$, we conclude that

$$
\lambda_{\Omega}\left(z_{t}\right) \leqslant \frac{\pi / 4}{\left|z_{t}-a\right|\left(k_{0}+|\log | \frac{z_{t}-a}{b-a}||\right)} \leqslant \frac{\pi / 4}{\frac{1}{\sqrt{2}}|\eta(t)-a|\left(k_{0}+\left|\log \frac{1}{\sqrt{2}}\right| \frac{\eta(t)-a}{b-a}||\right)}
$$

Next we note that $|\log (u / v)|=|\log u-\log v| \geqslant|\log u|-\log v$ for $u>0, v \geqslant 1$. Thus we continue the previous estimate by

$$
\lambda_{\Omega}\left(z_{t}\right) \leqslant \frac{2^{-3 / 2} \pi}{|\eta(t)-a|\left(k_{0}+|\log | \frac{\eta(t)-a}{b-a}||\right)} \cdot \frac{k_{0}+|\log | \frac{\eta(t)-a}{b-a}||}{k_{0}+|\log | \frac{\eta(t)-a}{b-a}||-\log \sqrt{2}} .
$$

Next we note that the second factor on the right hand side is estimated by $\frac{k_{0}}{k_{0}-\log \sqrt{2}}=\frac{c_{0}}{\pi}$. We denote $\Omega_{0}:=\mathbb{C} \backslash\{a, b\}$ and observe that $|\eta(t)-a| \geqslant \delta_{\Omega_{0}}(\eta(t))$. Hence

$$
\lambda_{\Omega}\left(z_{t}\right) \leqslant \frac{2^{-3 / 2} c_{0}}{|\eta(t)-a|\left(k_{0}+|\log | \frac{\eta(t)-a}{b-a}||\right)} \leqslant c_{0} \lambda_{\Omega_{0}}(\eta(t)) \leqslant c_{0} \lambda_{\Omega}(\eta(t)),
$$


since $\Omega \subseteq \Omega_{0}$. Because $\eta(t)$ and $z_{t}$ are parameterized by Euclidean arc-length, this inequality gives the result.

LEMma 3.4. Let us consider a Denjoy domain $\Omega, z_{0} \in \Omega \cap \overline{\mathbb{H}}$, a curve $\eta$ with Euclidean length $r$ starting at $z_{0}$, and $\sigma:=\left[z_{0}, z_{0}+i r\right]$. Then $\ell_{k, \Omega}(\sigma) \leqslant \sqrt{2} \ell_{k, \Omega}(\eta)$.

Proof. Using the notation of the previous proof and (3.1) we derive the estimate

$$
\frac{1}{\delta_{\Omega}\left(z_{t}\right)}=\frac{1}{\left|z_{t}-a\right|} \leqslant \frac{\sqrt{2}}{|\eta(t)-a|} \leqslant \frac{\sqrt{2}}{\delta_{\Omega}(\eta(t))}
$$

of the densities, from which the claim follows as before.

Definition 3.5. A function between two metric spaces $f: X \longrightarrow Y$ is an $(a, b)$-quasiisometry, $a \geqslant 1, b \geqslant 0$, if

$$
\frac{1}{a} d_{X}\left(x_{1}, x_{2}\right)-b \leqslant d_{Y}\left(f\left(x_{1}\right), f\left(x_{2}\right)\right) \leqslant a d_{X}\left(x_{1}, x_{2}\right)+b, \quad \text { for every } x_{1}, x_{2} \in X .
$$

If there exists a constant $c$ such that $d_{Y}(f(X), y) \leqslant c$ for every $y \in Y$, we say that $X$ and $Y$ are quasi-isometric.

An $(a, b)$-quasigeodesic in $X$ is an $(a, b)$-quasi-isometry between an interval of $\mathbb{R}$ and $X$.

The following lemma is easily proved directly from the definition, see [13, Lemma 4.4] if necessary.

Lemma 3.6. Let us consider a geodesic metric space $X$, an $(a, b)$-quasigeodesic $\gamma: I \longrightarrow$ $X$, with $I$ any interval, and $g: I \longrightarrow X$, with $d(g(t), \gamma(t)) \leqslant \varepsilon$ for every $t \in I$. Then $g$ is an $(a, b+2 \varepsilon)$-quasigeodesic.

Definition 3.7. Rays of the type $\{z \in \overline{\mathbb{H}} \cap \Omega: \operatorname{Re} z=c\}, c \in \mathbb{R}$, are called $A$-lines .

Halves of squares of the type $([a, a+i r] \cup[a+i r, a+2 r+i r] \cup[a+2 r+i r, a+2 r]) \cap \Omega, a \in$ $\mathbb{R}, r>0$, are called $B$-lines.

Halfcircles of the type $\left\{z \in \overline{\mathbb{H}} \cap \Omega:\left|z-x_{0}\right|=r\right\}, x_{0} \in \mathbb{R}, r>0$, are called $C$-lines.

The following result says that there are universal constants $a$ and $b$ such that the geodesics in $\mathbb{H}$ (the $A$-lines and the $C$-lines) are $(a, b)$-quasigeodesics in every Denjoy domain $\Omega$. For technical reasons it is often more convenient to work with $B$-lines, which are also shown to be quasigeodesics.

Theorem 3.8. Let us consider any Denjoy domain $\Omega$ and denote $c_{0}:=\frac{\pi k_{0}}{k_{0}-\log \sqrt{2}}<4$. Then

(i) A-lines are $\left(c_{0}, 0\right)$-quasigeodesics, $B$-lines are $\left(c_{0}, 2\right)$-quasigeodesics, and $C$-lines are $\left(c_{0}, 4\right)$-quasigeodesics of the hyperbolic metric.

(ii) $A$-lines are $(\sqrt{2}, 0)$-quasigeodesics, $B$-lines are $(\sqrt{2}, 2)$-quasigeodesics, and $C$-lines are $(\sqrt{2}, 4)$-quasigeodesics of the quasihyperbolic metric. 
Proof. We deal first with the hyperbolic metric. Consider a curve $\sigma$, which is either an $A$-line, a $B$-line or a $C$-line, parameterized by hyperbolic arc-length. Then

$$
h_{\Omega}(\sigma(s), \sigma(t)) \leqslant \ell_{h, \Omega}\left(\left.\sigma\right|_{[s, t]}\right)=t-s
$$

for every $s<t$ in the domain of $\sigma$. In order to obtain the other inequality, we deal with each case separately.

Assume first that $\sigma$ is an $A$-line. Let us consider a hyperbolic geodesic $\eta$ joining $\sigma(s)$ and $\sigma(t)$. Without loss of generality we can assume that $\operatorname{Im} \sigma(s)<\operatorname{Im} \sigma(t)$. Since the graph of $\sigma$ is a straight line, $\ell_{\text {Eucl }}\left(\left.\sigma\right|_{[s, t]}\right) \leqslant \ell_{\text {Eucl }}(\eta)$, and we can denote by $\eta_{0}$ the subcurve of $\eta$ starting at $\sigma(s)$ with $\ell_{\text {Eucl }}\left(\eta_{0}\right)=\ell_{\text {Eucl }}\left(\left.\sigma\right|_{[s, t]}\right)$. Applying Lemma 3.3 we deduce that

$$
t-s=\ell_{h, \Omega}\left(\left.\sigma\right|_{[s, t]}\right) \leqslant c_{0} \ell_{h, \Omega}\left(\eta_{0}\right) \leqslant c_{0} \ell_{h, \Omega}(\eta)=c_{0} h_{\Omega}(\sigma(s), \sigma(t)) .
$$

This proves the first statement.

Assume now that $\sigma$ is the $B$-line joining the points $a$ and $a+2 r$. Consider $s<t$ in the domain of $\sigma$. If $\sigma(s)$ and $\sigma(t)$ are both either in $[a, a+i r]$ or in $[a+2 r+i r, a+2 r]$, we can use the first case. Without loss of generality we can assume that $\sigma(s) \in[a, a+i r]$ and $\sigma(t) \in$ $[a+2 r+i r, a+2 r]$, since the other cases are easier.

Consider a hyperbolic geodesic $\eta$ joining $\sigma(s)$ and $\sigma(t)$. Denote by $\eta_{1}$ the subcurve of $\eta$ starting at $\sigma(s)$ with $\ell_{\text {Eucl }}\left(\eta_{1}\right)=r-\operatorname{Im} \sigma(s)$, and by $\eta_{2}$ the subcurve of $\eta$ ending at $\sigma(t)$ with $\ell_{\text {Eucl }}\left(\eta_{2}\right)=r-\operatorname{Im} \sigma(t)$. Since the Euclidean length of $\eta$ is at least $2 r, \eta_{1}$ and $\eta_{2}$ are disjoint. Applying Lemma 3.3 twice we deduce

$$
\begin{aligned}
\ell_{h, \Omega}([\sigma(s), a+i r])+\ell_{h, \Omega}([a+2 r+i r, \sigma(t)]) & \leqslant c_{0} \ell_{h, \Omega}\left(\eta_{1}\right)+c_{0} \ell_{h, \Omega}\left(\eta_{2}\right) \\
& \leqslant c_{0} \ell_{h, \Omega}(\eta)=c_{0} h_{\Omega}(\sigma(s), \sigma(t)) .
\end{aligned}
$$

In addition,

$$
\ell_{h, \Omega}([a+i r, a+2 r+i r]) \leqslant \ell_{h, \mathbb{H}^{2}}([a+i r, a+2 r+i r])=\int_{a}^{a+2 r} \frac{d x}{r}=2,
$$

and hence

$$
\begin{aligned}
t-s & =\ell_{h, \Omega}([\sigma(s), a+i r])+\ell_{h, \Omega}([a+2 r+i r, \sigma(t)])+\ell_{h, \Omega}([a+i r, a+2 r+i r]) \\
& \leqslant c_{0} h_{\Omega}(\sigma(s), \sigma(t))+2 .
\end{aligned}
$$

This proves the second statement.

Suppose finally that $\sigma$ is a $C$-line, with center $a+r$ and radius $r$. Let $\sigma^{\prime}$ be the $B$-line from the previous case. For a point $z \in \sigma$, we choose a corresponding point $z^{\prime} \in \sigma^{\prime}$ such that $\operatorname{Im}(z)=$ $\operatorname{Im}\left(z^{\prime}\right)$ and $\left|z-z^{\prime}\right| \leqslant \operatorname{Im}(z)$. Then $h_{\Omega}\left(z, z^{\prime}\right) \leqslant h_{\mathbb{H}}\left(z, z^{\prime}\right) \leqslant h_{\mathbb{H}}(i, i+1)<1$ and we conclude by Lemma 3.6 that the $C$-line is a $\left(c_{0}, 4\right)$-quasigeodesic.

The proof for the quasihyperbolic metric is the same, replacing $c_{0}$ by $\sqrt{2}$ and using Lemma 3.4 instead of Lemma 3.3.

Since $\overline{\mathbb{H}} \cap \Omega$ is $\delta$-hyperbolic for $\delta$ a universal constant (see [13, Lemma 3.1]), by geodesic stability the geodesics in $\overline{\mathbb{H}} \cap \Omega$ are located at Hausdorff distance less than $c$ (a universal constant) from quasigeodesics in $\overline{\mathbb{H}} \cap \Omega$. Therefore, the previous theorem gives precise information about the geodesics in every Denjoy domain. These statements can be made precise using the following definitions and results.

Definition 3.9. Let us consider $H>0$, a metric space $(X, d)$, and subsets $Y, Z \subseteq X$. The set $V_{H}(Y):=\{x \in X: d(x, Y) \leqslant H\}$ is called the $H$-neighborhood of $Y$ in $X$. The Hausdorff distance of $Y$ to $Z$ is defined by $\mathcal{H}_{d}(Y, Z):=\inf \left\{H>0: Y \subseteq V_{H}(Z), Z \subseteq V_{H}(Y)\right\}$. 
TheOREM 3.10 [10, p. 87]. For each $\delta \geqslant 0, a \geqslant 1$ and $b \geqslant 0$, there exists a constant $H=$ $H(\delta, a, b)$ with the following property:

Let $(X, d)$ be a $\delta$-hyperbolic geodesic metric space and let $g$ be a $(a, b)$-quasigeodesic joining $x$ and $y$. If $\gamma$ is a geodesic joining $x$ and $y$, then $\mathcal{H}_{d}(g, \gamma) \leqslant H$.

This property is known as geodesic stability. M. Bonk has proved that, in fact, geodesic stability is equivalent to Gromov hyperbolicity [5].

Now we can prove in a simple way the following unexpected result.

TheOREM 3.11. There exists a universal constant $H_{0}$ such that $\mathcal{H}_{h, \Omega}\left(\gamma_{h}, \gamma_{k}\right) \leqslant H_{0}$ for every Denjoy domain $\Omega$, every $z, w \in \Omega \cap \overline{\mathbb{H}}$ and every pair of geodesics $\gamma_{h}$ and $\gamma_{k}$ of the hyperbolic and the quasihyperbolic metrics of $\Omega$ joining $z$ and $w$.

Proof. By [13, Lemma 3.1], there exists a universal constant $\delta$ such that for any Denjoy domain $\Omega$, the geodesic space $\Omega \cap \overline{\mathbb{H}}$ is $\delta$-hyperbolic (for both the hyperbolic and the quasihyperbolic metrics).

Consider either the $A$-line or the $C$-line $g$ joining $z, w \in \Omega \cap \overline{\mathbb{H}}$. By Theorem 3.8 there exist universal constants $a, b$ such that $g$ is an $(a, b)$-quasigeodesic for both the hyperbolic and the quasihyperbolic metrics.

Hence, by Theorem 3.10 there exist universal constants $H_{h}, H_{k}$ such that $\mathcal{H}_{h, \Omega}\left(\gamma_{h}, g\right) \leqslant H_{h}$ and $\mathcal{H}_{k, \Omega}\left(\gamma_{k}, g\right) \leqslant H_{k}$. Since $h_{\Omega} \leqslant 2 k_{\Omega}$, we deduce

$$
\mathcal{H}_{h, \Omega}\left(\gamma_{h}, \gamma_{k}\right) \leqslant \mathcal{H}_{h, \Omega}\left(\gamma_{h}, g\right)+\mathcal{H}_{h, \Omega}\left(\gamma_{k}, g\right) \leqslant \mathcal{H}_{h, \Omega}\left(\gamma_{h}, g\right)+2 \mathcal{H}_{k, \Omega}\left(\gamma_{k}, g\right) \leqslant H_{h}+2 H_{k}
$$

as claimed.

\section{Equivalence of the hyperbolic and the quasihyperbolic metrics}

It is well-known that if two geodesic metric spaces are quasi-isometric, then either both are Gromov hyperbolic or neither is. Beardon's and Pommerenke's result, quoted as Theorem 3.2, implies that there is not in general a constant such that $k_{\Omega} \leqslant c h_{\Omega}$, whereas the upper bound $h_{\Omega} \leqslant 2 k_{\Omega}$ always holds. Therefore it is quite surprising that $h_{\Omega}$ is Gromov hyperbolic if and only if $k_{\Omega}$ is when $\Omega$ is Denjoy domain, which we now proceed to show. It remains an open question whether this type of equivalence holds in more general domains.

The next result was proven for the hyperbolic metric in [1, Theorem 5.1]. The equivalence among (1), (2) and (3) for the quasihyperbolic metric was delt with in [13, Theorem 3.3]. The equivalence between each of them and (4) can be obtained with the same argument as in the proof of [1, Theorem 5.1]. A fundamental quasigeodesic is defined analogously to a fundamental geodesic.

Theorem 4.1. Let $\Omega$ be a Denjoy domain and denote by $\kappa_{\Omega}$ the Poincaré or quasihyperbolic metric in $\Omega$. Fix $\alpha \geqslant 1$ and $\beta \geqslant 0$. Then the following conditions are quantitatively equivalent:

(1) $\kappa_{\Omega}$ is $\delta$-hyperbolic.

(2) There exists a constant $c_{1}$ such that $\kappa_{\Omega}(z, \mathbb{R}) \leqslant c_{1}$ for every $z$ belonging to a fundamental geodesic.

(3) There exists a constant $c_{2}$ such that for a fixed choice of fundamental geodesics $\left\{\gamma_{n}\right\}_{n=1}^{\infty}$ we have $\kappa_{\Omega}(z, \mathbb{R}) \leqslant c_{2}$ for every $z \in \cup_{n} \geqslant 1 \gamma_{n}$.

(4) There exists a constant $c_{3}$ such that for a fixed choice of fundamental $(\alpha, \beta)$ quasigeodesics $\left\{g_{n}\right\}_{n=1}^{\infty}$ we have $\kappa_{\Omega}(z, \mathbb{R}) \leqslant c_{3}$ for every $z \in \cup_{n} \geqslant 1 g_{n}$. 
This result has the following Corollary that we will use later. By a fundamental quasigeodesic $g_{m n}$ we mean a quasigeodesic in the upper half plane connecting $\left(a_{m}, b_{m}\right)$ and $\left(a_{n}, b_{n}\right)$.

Corollary 4.2. Let $\Omega$ be a Denjoy domain $\Omega \cap \mathbb{R}=\cup_{n \geqslant 0}\left(a_{n}, b_{n}\right)$ and denote by $\kappa_{\Omega}$ the Poincaré or quasihyperbolic metric in $\Omega$. Fix $\alpha \geqslant 1$ and $\beta \geqslant 0$. Then the following conditions are quantitatively equivalent:

(1) $\kappa_{\Omega}$ is $\delta$-hyperbolic.

(2) There exists a constant $c$ such that for a fixed choice of fundamental $(\alpha, \beta)$ quasigeodesics $\left\{g_{m n}\right\}_{m \neq n}$ we have $\kappa_{\Omega}(z, \mathbb{R}) \leqslant c$ for every $z \in \cup_{m \neq n} g_{m n}$.

Proof. Assume first that (2) holds. Then, taking $m=0$, we obtain condition (4) in Theorem 4.1, and we can conclude that $\kappa_{\Omega}$ is $\delta$-hyperbolic.

Assume now that (1) holds. By Theorem 4.1 there exists a constant $c$ such that $\kappa_{\Omega}(z, \mathbb{R}) \leqslant c$ for every $z$ lying on an $(\alpha, \beta)$-quasigeodesics joining the intervals 0 and $n$. However, there is nothing special about the interval 0 : we can relabel the intervals so that interval $m$ is numbered 0 , and thus $\kappa_{\Omega}(z, \mathbb{R}) \leqslant c$ for every $z \in \cup_{m \neq n} g_{m n}$.

Now we can give a first characterization of Gromov hyperbolicity of Denjoy domains, which is still stated in terms of the metric itself.

Theorem 4.3. Let $\Omega$ be a Denjoy domain with $\Omega \cap \mathbb{R}=\cup_{n=0}^{\infty}\left(a_{n}, b_{n}\right)$, and denote by $\kappa_{\Omega}$ the Poincaré or quasihyperbolic metric in $\Omega$. Let us fix $x_{n} \in\left(a_{n}, b_{n}\right)$ for each $n \geqslant 0$. Then $\kappa_{\Omega}$ is Gromov hyperbolic if and only if

$$
\sup _{n \geqslant 0} \sup _{y \in(0, R)} \kappa_{\Omega}\left(x_{n}+i y, \mathbb{R}\right)<\infty
$$

quantitatively, where $R:=\sup \left|x_{m}-x_{n}\right|$.

Proof. Suppose first that $\Omega$ is Gromov hyperbolic. Fix $n$ and choose $m$ so that $\left|x_{n}-x_{m}\right| \geqslant$ $\frac{1}{3} R$. Let $g_{m n}$ be the $B$-line joining $x_{m}$ with $x_{n}$. Then

$$
\begin{aligned}
\sup _{y \in(0, R)} \kappa_{\Omega}\left(x_{n}+i y, \mathbb{R}\right) & \leqslant \sup _{y \in\left(0, \frac{1}{6} R\right)} \kappa_{\Omega}\left(x_{n}+i y, \mathbb{R}\right)+h_{\mathbb{H}}\left(x_{n}+\frac{i}{6} R, x_{n}+i R\right) \\
& \leqslant \sup _{z \in g_{m n}} \kappa_{\Omega}(z, \mathbb{R})+\log 6<C
\end{aligned}
$$

by Corollary 4.2 since $g_{m n}$ is a quasigeodesic by Theorem 3.8. Taking the supremum over $n$ gives one implication of the theorem.

To prove the opposite implication, we assume that the inequality in the theorem holds. Let $g_{m n}$ be a $B$-line joining $x_{m} \in\left(a_{m}, b_{m}\right)$ with $x_{n} \in\left(a_{n}, b_{n}\right)$. Note first that

$$
\ell_{\kappa, \Omega}\left(\left[x_{m}+i r, x_{n}+i r\right]\right) \leqslant 2 \ell_{k, \mathbb{H}}\left(\left[x_{m}+i r, x_{n}+i r\right]\right)=4,
$$

where $r=\frac{\left|x_{n}-x_{m}\right|}{2}$. Thus the horizontal part of the $B$-line has lenght at most 4 . If $z \in g_{m n}$ does not belong to the horizontal part, then $z=x_{n}+i y$ or $z=x_{m}+i y$ for some $y \in(0, R)$. Hence

$$
\sup _{z \in g_{m n}} \kappa_{\Omega}(z, \mathbb{R}) \leqslant \sup _{y \in(0, R)}\left(\kappa_{\Omega}\left(x_{n}+i y, \mathbb{R}\right)+\kappa_{\Omega}\left(x_{m}+i y, \mathbb{R}\right)\right)+2<C .
$$

Since this inequality holds for every $B$-line, the second condition of Corollary 4.2 is satisfied, and we conclude that $\Omega$ is Gromov hyperbolic. 
REMARK 4.4. If $\Omega$ in the previous theorem is such that $\lim _{\sup } \rightarrow \infty\left|a_{n}\right|=\infty$, then $R=\infty$ and the supremum is taken over all $y \in(0, \infty)$.

We can now deduce the main theorem of the paper. For this we need the following well-known result, for a proof see e.g. [13, Lemma 4.3].

Lemma 4.5. Let $\gamma$ be a curve in a domain $\Omega \subset \mathbb{R}^{n}$. Then $\ell_{k, \Omega}(\gamma) \geqslant \log \left(1+\frac{\ell_{\text {Eucl }}(\gamma)}{\delta_{\Omega}(\gamma(0))}\right)$.

Theorem 4.6. Let $\Omega$ be a Denjoy domain. Then $k_{\Omega}$ is Gromov hyperbolic if and only if $h_{\Omega}$ is, quantitively.

Proof. Fix $x_{n} \in\left(a_{n}, b_{n}\right)$ for each $n \geqslant 0$ and let $R$ be as in Theorem 4.3. Suppose first that $k_{\Omega}$ is Gromov hyperbolic. Using Theorem 4.3 and the inequality $h_{\Omega} \leqslant 2 k_{\Omega}$ we conclude that

$$
\sup _{n \geqslant 0} \sup _{y \in(0, R)} h_{\Omega}\left(x_{n}+i y, \mathbb{R}\right) \leqslant 2 \sup _{n \geqslant 0} \sup _{y \in(0, R)} k_{\Omega}\left(x_{n}+i y, \mathbb{R}\right)<\infty .
$$

Therefore, by the same theorem, $h_{\Omega}$ is Gromov hyperbolic.

Assume next that $k_{\Omega}$ is not Gromov hyperbolic. By Theorem 4.3 we can choose $y \in(0, R)$ and $n$ so that $k_{\Omega}\left(x_{n}+i y, \mathbb{R}\right)>M^{2} \gg 1$. After a translation and dilatation, we may assume that $x_{n}=0$ and $y=1$. Consider the Euclidean disk $B=B(0, M)$. Suppose $(a-r, a+r) \subset B \cap \Omega$, for some $a \in \Omega \cap \mathbb{R}, r>0$. Then

$$
\begin{aligned}
k_{\Omega}(i, a) & \leqslant \ell_{k, \Omega}([i, a+i])+\ell_{k, \Omega}([a+i, a]) \\
& \leqslant \ell_{k, \mathbb{H}}([i, a+i])+\ell_{k, \mathbb{C} \backslash\{a+r\}}([a+i, a]) \\
& =a+\operatorname{ar} \sinh \frac{1}{r} \leqslant M+\log \left(1+\frac{2}{r}\right) .
\end{aligned}
$$

Since $k_{\Omega}(i, a) \geqslant k_{\Omega}(i, \mathbb{R})>M^{2}$, we conclude that

$$
r<\frac{2}{\exp \left(M^{2}-M\right)-1}=: \delta .
$$

Therefore, there exists no open interval in $B \cap \Omega \cap \mathbb{R}$ of size greater than $2 \delta$.

Let $\gamma$ be a path from $i$ to $\mathbb{R}$. Let $\gamma^{\prime}$ be the part of $\gamma$ which connects $i$ to $\partial B \cup(\mathbb{R}+i \delta)$. Note that $\gamma^{\prime}$ stays everywhere at distance at least $\delta$ from the real axis. Since there are no gaps in the boundary $\partial \Omega$ of size greater than $2 \delta$ in $B$, we conclude that $\beta_{\Omega} \leqslant \log 2$ for every point on the path. Thus $\ell_{h, \Omega}\left(\gamma^{\prime}\right) \geqslant c \ell_{k, \Omega}\left(\gamma^{\prime}\right)$ with constant independend of $M$. If $\gamma^{\prime}$ hits $\partial B$, then $\ell_{\text {Eucl }}\left(\gamma^{\prime}\right) \geqslant M-1$ and $\delta_{\Omega}(i) \leqslant 1+\delta$ so that $\ell_{k, \Omega}\left(\gamma^{\prime}\right) \geqslant \log \left(1+\frac{M-1}{1+\delta}\right)$, by Lemma 4.5. If $\gamma^{\prime}$ hits $\mathbb{R}+i \delta$ at $z$, then $\ell_{\text {Eucl }}\left(\gamma^{\prime}\right) \geqslant 1-\delta$ and $\delta_{\Omega}(z) \leqslant 2 \delta$ so that $\ell_{k, \Omega}\left(\gamma^{\prime}\right) \geqslant \log \left(1+\frac{1-\delta}{2 \delta}\right)$, again by Lemma 4.5. In conclusion,

$$
h_{\Omega}(i, \mathbb{R}) \geqslant \inf _{\gamma} \ell_{h, \Omega}\left(\gamma^{\prime}\right) \geqslant c \min \left\{\log \left(\frac{1}{2}(M-1)\right), \log \left(\frac{1}{2}\left(1+\delta^{-1}\right)\right\}=c \log \left(\frac{1}{2}(M-1)\right),\right.
$$

since $\delta^{-1}=\frac{1}{2} \exp \left(M^{2}-M\right)-1>M^{2}$. But $M$ can be chosen arbitrarily large, so this implies that $\sup _{n \geqslant 0} \sup _{y \in(0, R)} h_{\Omega}\left(x_{n}+i y, \mathbb{R}\right)=\infty$, and hence $h_{\Omega}$ is not Gromov hyperbolic, by Theorem 4.3.

\section{Concrete results}

In Theorem 4.3 we gave a characterization of when a Denjoy domain is Gromov hyperbolic with the hyperbolic or quasihyperbolic metric. However, this characterization was in terms 
of the metrics themselves. In this section we give a characterization based only on Euclidean distances and provide some concrete applications of this result. Since our characterization applies only in the case of Denjoy domains, it is more concrete and easier to use than the general characterization of Z. Balogh and S. Buckley [2].

Recall that the $j_{\Omega}$ metric is defined as

$$
j_{\Omega}(x, y):=\log \left(1+\frac{|x-y|}{\min \left\{\delta_{\Omega}(x), \delta_{\Omega}(y)\right\}}\right),
$$

cf. $[\mathbf{9}, \mathbf{2 4}]$. A uniform path is a continuous mapping $\gamma:[0, a] \rightarrow \Omega$ parametrized by arc-length such that

$$
|\gamma(0)-\gamma(a)| \leqslant c a \quad \text { and } \min \{t, a-t\} \leqslant c \delta_{\Omega}(\gamma(t)) \quad \forall t \in(0, a) .
$$

By integrating the density $\frac{1}{\delta_{\Omega}(z)}$ of the quasihyperbolic metric over the uniform path we easily find that $k_{\Omega}(\gamma(0), \gamma(a)) \leqslant c j_{\Omega}(\gamma(0), \gamma(a))$. Since $j_{\Omega}$ is the inner metric of $k_{\Omega}$, the opposite inequality always holds, with constant 1 .

If $\Omega$ is a Denjoy domain and $x, y \in \mathbb{H}$, then the points can be joined by a uniform path in $\Omega$, namely an arc of the circle centered on $\mathbb{R}$ which contains $x$ and $y$. Therefore we have the following result:

LEmma 5.1. There exists a universal constant $c$ such that for every Denjoy domain $\Omega$ and $x, y$ in the upper half-plane we have

$$
j_{\Omega}(x, y) \leqslant k_{\Omega}(x, y) \leqslant c j_{\Omega}(x, y) .
$$

Now we are ready for the Euclidean distance version of Theorem 4.3. For brevity we use the following notation: we say that $A \lesssim B$, where $A, B$ depend on some parameters, if there exists a constant $c$ such that $A \leqslant c B$ for every value of the parameters. We say that $A \approx B$ if $A \lesssim B \lesssim A$. We also use the convention that $\min \{t, \infty\}=t$.

TheOREm 5.2. Let $\Omega$ be a Denjoy domain with $\Omega \cap \mathbb{R}=\cup_{n=0}^{\infty}\left(a_{n}, b_{n}\right)$ and $x_{n} \in\left(a_{n}, b_{n}\right)$ for every $n \geqslant 0$ and let $R$ be as in Theorem 4.3. Then $\kappa_{\Omega}$ is Gromov hyperbolic (quantitatively) if and only if for every $n \geqslant 0$ and every $y \in(0, R)$ there exists $m \geqslant 0$ and $x \in\left(a_{m}, b_{m}\right)$ such that $\left|x_{n}-x\right| \lesssim y \lesssim \min \left\{b_{m}-x, x-a_{m}\right\}$.

Proof. By Theorem 4.6 it suffices to consider the quasihyperbolic metric. By Theorem 4.3 and Lemma 5.1, $k_{\Omega}$ is Gromov hyperbolic if and only if

$$
\sup _{n \geqslant 0} \sup _{y \in(0, R)} j_{\Omega}\left(x_{n}+i y, \mathbb{R}\right)<\infty
$$

which, again, holds if and only if

$$
\begin{aligned}
\sup _{n \geqslant 0} & \sup _{y \in(0, R)} \inf _{m \geqslant 0} \inf _{x \in\left(a_{m}, b_{m}\right)} \frac{\left|x_{n}+i y-x\right|}{\min \left\{\delta_{\Omega}\left(x_{n}+i y\right), \delta_{\Omega}(x)\right\}} \\
& \approx \sup _{n \geqslant 0} \sup _{y \in(0, R)} \inf _{m \geqslant 0} \inf _{x \in\left(a_{m}, b_{m}\right)} \frac{\left|x_{n}-x\right|+y}{\min \left\{d_{n}, b_{m}-x, x-a_{m}\right\}}<\infty,
\end{aligned}
$$

where $d_{n}:=\delta_{\Omega}\left(x_{n}+i y\right) \approx y+\min \left\{b_{n}-x_{n}, x_{n}-a_{n}\right\}$. This inequality holds if and only if there exists $c>0$ satisfying the following property: for every $n$ and every $y \in(0, R)$ there exists $m \geqslant 0$ and $x \in\left(a_{m}, b_{m}\right)$ such that

$$
\frac{\left|x_{n}-x\right|}{\min \left\{d_{n}, b_{m}-x, x-a_{m}\right\}}+\frac{y}{d_{n}}+\frac{y}{\min \left\{b_{m}-x, x-a_{m}\right\}}<c .
$$


Note that the second term is at most 1 , and so it can be dropped. If $\left|x_{n}-x\right| \lesssim y \lesssim \min \left\{b_{m}-\right.$ $\left.x, x-a_{m}\right\}$, then (5.2) certainly holds; this proves one of the implications.

For the opposite implication assume that (5.2) holds and consider two cases. First, if $y \leqslant$ $b_{n}-a_{n}$, then we choose $m=n$. We assume that $b_{n}-x_{n} \geqslant x_{n}-a_{n}$; the other case is similar. Then we choose $x=x_{n}+\frac{y}{4} \in\left(a_{n}, b_{n}\right)$. Obviously, $\left|x_{n}-x\right| \lesssim y$. Also,

$$
\min \left\{b_{n}-x, x-a_{n}\right\}=\min \left\{b_{n}-x_{n}-\frac{y}{4}, x_{n}+\frac{y}{4}-a_{n}\right\} \geqslant \min \left\{\frac{b_{n}-a_{n}}{2}-\frac{y}{4}, \frac{y}{4}\right\}=\frac{y}{4} .
$$

Thus $\left|x_{n}-x\right| \lesssim y \lesssim \min \left\{b_{m}-x, x-a_{m}\right\}$ in this case.

Then we deal with the main case, $y>b_{n}-a_{n}$. Separating the third term in (5.2) yields the inequality $y \lesssim \min \left\{b_{m}-x, x-a_{m}\right\}$. The boundedness of the first term in (5.2) implies that $\left|x_{n}-x\right| \lesssim \min \left\{d_{n}, b_{m}-x, x-a_{m}\right\}$, hence in particular $\left|x_{n}-x\right| \lesssim d_{n} \approx y+\min \left\{b_{n}-\right.$ $\left.x_{n}, x_{n}-a_{n}\right\} \leqslant 2 y$.

Let us state now some applications of our characterization.

Lemma 5.3. Let $\Omega$ be a Denjoy domain with $\Omega \cap \mathbb{R}=\cup_{n=0}^{\infty}\left(a_{n}, b_{n}\right)$ and $b_{0}>0$. Suppose that $a_{n+1} \geqslant b_{n} \geqslant K a_{n}$ and $a_{n} \leqslant L b_{n-1}$ for fixed $K, L>1$, and every $n \geqslant 1$. Then $h_{\Omega}$ and $k_{\Omega}$ are Gromov hyperbolic, quantitatively.

Proof. The case $a_{0}=-\infty$ has been considered in [13, Theorem 3.6] so we may assume here that $a_{0}=0$. Choose $x_{n}:=\left(a_{n}+b_{n}\right) / 2$ for each $n$. Fix $n \geqslant 0$ and $y>0$. If $y<x_{n}$, then we chose $m=n$ and $x=x_{n}$ and easily see that $\left|x_{n}-x\right| \lesssim y \lesssim \min \left\{b_{m}-x, x-a_{m}\right\}$ holds. If $y \geqslant x_{n}$ we choose $m \geqslant n$ such that $a_{m} \leqslant y<a_{m+1}$ and set $x=\min \left\{x_{m}, \frac{K+1}{2} y\right\}$. Note that $\frac{K+1}{2} a_{m} \leqslant x \leqslant x_{m}$ so that $x \in\left(a_{m}, b_{m}\right)$.

It follows from the choice of $x$ that $\left|x_{n}-x\right|=x-x_{n} \leqslant \frac{K+1}{2} y$, so $\left|x_{n}-x\right| \lesssim y$. For the second condition from Theorem 5.2, $y \lesssim \min \left\{b_{m}-x, x-a_{m}\right\}$, we consider two cases: if $x=x_{m}$, then

$$
y<a_{m+1} \leqslant L b_{m} \leqslant \frac{L K}{K-1}\left(b_{m}-a_{m}\right)=\frac{2 L K}{K-1} \min \left\{b_{m}-x, x-a_{m}\right\} ;
$$

if $x=\frac{K+1}{2} y<x_{m}$, then $\min \left\{b_{m}-x, x-a_{m}\right\}=x-a_{m} \geqslant \frac{K+1}{2} y-y=\frac{K-1}{2} y \gtrsim y$. Therefore the inequality $\left|x_{n}-x\right| \lesssim y \lesssim \min \left\{b_{m}-x, x-a_{m}\right\}$ holds in both cases, and the claim follows from Theorem 5.2.

Lemma 5.4. Let $\Omega$ be a Denjoy domain with $\Omega \cap \mathbb{R}=\cup_{n=0}^{\infty}\left(a_{n}, b_{n}\right), a_{0}>-\infty, b_{0}>0$ and $b_{n} \leqslant a_{n+1}$ for every $n$. If $\lim \sup _{n \rightarrow \infty} a_{n+1} / b_{n}=\infty$, then $h_{\Omega}$ and $k_{\Omega}$ are not Gromov hyperbolic.

Proof. Without loss of generality we can assume that $a_{0}=-b_{0}$ and $x_{0}=0$. For $M \gg 1$ we choose $k \geqslant 0$ such that $a_{k+1} / b_{k} \geqslant M^{2}$. Choose $n=0$ and $y=M b_{k}$ in Theorem 5.2. Then $\left|x-x_{n}\right| / y \geqslant a_{k+1} /\left(M b_{k}\right) \geqslant M$ for $m>k$ and $x \in\left(a_{m}, b_{m}\right)$. On the other hand, $\min \left\{b_{m}-\right.$ $\left.x, x-a_{m}\right\} \leqslant b_{m}-a_{m} \leqslant b_{k}=\frac{1}{M} y$ for $m \leqslant k$. Since $M$ can be arbitrarily large, we see that the inequalities $\left|x_{n}-x\right| \lesssim y \lesssim \min \left\{b_{m}-x, x-a_{m}\right\}$ do not both hold for any $m$. Therefore $h_{\Omega}$ and $k_{\Omega}$ are not Gromov hyperbolic, by Theorem 5.2.

We deduce the following result from Lemmas 5.3, 5.4 and [13, Theorem 1.1].

Theorem 5.5. Let $\Omega$ be a Denjoy domain with $\Omega \cap \mathbb{R}=\cup_{n=0}^{\infty}\left(a_{n}, b_{n}\right), b_{0}>0, b_{n} \leqslant a_{n+1}$ for every $n$, and $\liminf _{n \rightarrow \infty} b_{n} / a_{n}>1$.

(i) If $a_{0}=-\infty$, then $h_{\Omega}$ and $k_{\Omega}$ are Gromov hyperbolic. 
(ii) If $a_{0}>-\infty$, then $h_{\Omega}$ and $k_{\Omega}$ are Gromov hyperbolic if and only if

$$
\limsup _{n \rightarrow \infty} \frac{a_{n+1}}{b_{n}}<\infty .
$$

We conclude with some variants of the previous results, which are stronger, but more complicated to state.

Proposition 5.6. Let us consider a Denjoy domain $\Omega$ with $\Omega \cap \mathbb{R}=\cup_{n=0}^{\infty}\left(a_{n}, b_{n}\right)$ and $b_{n} \leqslant a_{n+1}$ for every $n$. If there exists a subsequence $\left\{n_{k}\right\}$ such that

$$
\lim _{k \rightarrow \infty} \frac{b_{n_{k}}-a_{n_{k}}}{a_{n_{k}}-b_{n_{k}-1}}=\lim _{k \rightarrow \infty} \frac{b_{n_{k}}-a_{n_{k}}}{a_{n_{k}+1}-b_{n_{k}}}=0,
$$

then the metrics $k_{\Omega}$ and $h_{\Omega}$ are not Gromov hyperbolic.

Proof. We choose $n_{k}$ so that both limits in the statement of the theorem are smaller than $M^{-2}$ for $M \gg 1$. Choosing $m=n_{k}$ and $y=M b_{n_{k}}$ and arguing as in Lemma 5.4 gives the result.

The argument in the proof of Lemma 5.4 and Proposition 5.6 gives the following stronger but more complicated results. Here we dispense with the hypothesis $b_{n} \leqslant a_{n+1}$; this allows, for instance, $\partial \Omega$ to contain Cantor sets.

Proposition 5.7. Let us consider a Denjoy domain $\Omega$ with $\left(-\infty, a_{0}\right],\left\{\left[u_{n}, v_{n}\right]\right\}_{n} \subset \mathbb{R} \backslash$ $\Omega, \lim _{n \rightarrow \infty} u_{n}=\infty$ and $\lim _{n \rightarrow \infty} v_{n} / u_{n}=\infty$. Then the metrics $k_{\Omega}$ and $h_{\Omega}$ are not Gromov hyperbolic.

Proposition 5.8. Let us consider a Denjoy domain $\Omega$ with $\left\{\left[u_{n}, v_{n}\right]\right\}_{n},\left\{\left[U_{n}, V_{n}\right]\right\}_{n} \subset \mathbb{R} \backslash$ $\Omega$ such that $\left(v_{n}, U_{n}\right) \cap \Omega \neq \emptyset$ for every $n$ and

$$
\lim _{n \rightarrow \infty} \frac{U_{n}-v_{n}}{v_{n}-u_{n}}=\lim _{n \rightarrow \infty} \frac{U_{n}-v_{n}}{V_{n}-U_{n}}=0,
$$

then the metrics $k_{\Omega}$ and $h_{\Omega}$ are not Gromov hyperbolic.

REMARK 5.9. Notice that we do not require $\left(v_{n}, U_{n}\right) \subset \Omega$; in fact, $\left(v_{n}, U_{n}\right) \cap \Omega$ can be any open set in $\left(v_{n}, U_{n}\right)$.

\section{References}

1. V. Alvarez, A. Portilla, J. M. Rodríguez and E. Tourís, 'Gromov hyperbolicity of Denjoy domains', Geom. Dedicata 121 (2006), 221-245.

2. Z. M. BALOGH and S. M. BuCKLeY, 'Geometric characterizations of Gromov hyperbolicity', Invent. Math. 153 (2003), 261-301.

3. A. F. Beardon and Ch. Pommerenke, 'The Poincaré metric of a plane domain', J. London Math. Soc. 18 (1978), 475-483.

4. Y. Benoist, 'Convexes hyperboliques et fonctions quasisymétriques', Publ. Math. Inst. Hautes Études Sci. 97 (2003), 181-237.

5. M. Bonk, 'Quasi-geodesic segments and Gromov hyperbolic spaces', Geom. Dedicata 62 (1996), 281-298.

6. M. Bonk, J. Heinonen, and P. Koskela, Uniformizing Gromov hyperbolic spaces, Astérisque 270 (2001).

7. M. Bonk and O. Schramm, 'Embeddings of Gromov hyperbolic spaces', Geom. Funct. Anal. 10 (2000), no. $2,266-306$. 
8. T. Foertsch and V. Schroeder, 'A product construction for hyperbolic metric spaces', Illinois J. Math. 49 (2005), no. 3, 793-810.

9. F. W. Gehring and B. P. Palka, 'Quasiconformally homogeneous domains', J. Anal. Math. 30 (1976), $172-199$.

10. E. Ghys and P. DE LA HARPe, Sur les Groupes Hyperboliques d'après Mikhael Gromov, Progress in Mathematics, Volume 83, Birkhäuser, 1990.

11. M. Gromov, Hyperbolic groups, Essays in group theory, 75-263, Math. Sci. Res. Inst. Publ., 8, Springer, New York, 1987.

12. P. A. Ḧ̈stö, 'Gromov hyperbolicity of the $j_{G}$ and $\tilde{\jmath}_{G}$ metrics', Proc. Amer. Math. Soc. 134 (2006), $1137-1142$

13. P. A. Hästö, H. Lindén, A. Portilla, J. M. Rodríguez and E. Tourís, 'Gromov hyperbolicity of Denjoy domains with hyperbolic and quasihyperbolic metrics', Preprint (2007).

14. A. Karlsson and G. A. Noskov, 'The Hilbert metric and Gromov hyperbolicity', Enseign. Math. 48 (2002), 73-89.

15. H. Lindén, 'Gromov hyperbolicity of certain conformal invariant metrics', Ann. Acad. Sci. Fenn. Math. 32 (2007), no. 1, 279-288.

16. A. Naor, Y. Peres, O. Schramm and S. Sheffield, 'Markov chains in smooth Banach spaces and Gromovhyperbolic metric spaces', Duke Math. J. 134 (2006), no. 1, 165-197.

17. A. Portilla, J. M. Rodríguez and E. Tourís, 'Gromov hyperbolicity through decomposition of metric spaces II', J. Geom. Anal. 14 (2004), 123-149.

18. A. Portilla, J. M. Rodríguez and E. Tourís, 'The topology of balls and Gromov hyperbolicity of Riemann surfaces', Diff. Geom. Appl. 21 (2004), 317-335.

19. A. Portilla, J. M. Rodríguez and E. Tourís, 'The role of funnels and punctures in the Gromov hyperbolicity of Riemann surfaces', Proc. Edinburgh Math. Soc. 49 (2006), 399-425.

20. A. Portilla, J. M. Rodríguez and E. Tourís, 'A real variable characterization of Gromov hyperbolicity of flute surfaces', Preprint (2007).

21. J. M. Rodríguez and E. Tourís, 'Gromov hyperbolicity through decomposition of metric spaces', Acta Math. Hung. 103 (2004), 53-84.

22. J. M. Rodríguez and E. Tourís, 'A new characterization of Gromov hyperbolicity for Riemann surfaces', Publ. Mat. 50 (2006), 249-278.

23. J. M. Rodríguez and E. Tourís, 'Gromov hyperbolicity of Riemann surfaces', Acta Math. Sinica 23 (2007), 209-228.

24. M. Vuorinen, 'Conformal invariants and quasiregular mappings', J. Anal. Math. 45 (1985), 69-115.

25. S. WENGER, 'Gromov hyperbolic spaces and the sharp isoperimetric constant', Invent. Math. 171 (2008), no. $1,227-255$.

Peter Hästö

Department of Mathematical Sciences

P.O. Box 3000

FI-90014 University of Oulu

Finland

peter.hasto@helsinki.fi

\author{
Ana Portilla \\ St. Louis University (Madrid Campus) \\ Avenida del Valle 34 \\ 28003 Madrid \\ Spain \\ portillaa@madrid.slu.edu
}

José M. Rodríguez and Eva Tourís

Departamento de Matemáticas

Universidad Carlos III de Madrid

Avenida de la Universidad 30

28911 Leganés, Madrid

Spain

jomaro@math.uc3m.es etouris@math.uc3m.es 